Internist 2010 · 51:313-313

DOI 10.1007/s00108-009-2572-y

Online publiziert: 21. Februar 2010

๑) Springer-Verlag 2010

\author{
S. Schellong ${ }^{1} \cdot$ A. Creutzig ${ }^{2}$ \\ ${ }^{1}$ Municipal Hospital Friedrichstadt, Dresden \\ ${ }^{2}$ Angiologische Praxis Luisenstraße und Klinikum \\ Oststadt-Heidehaus der Region Hannover
}

\title{
Erkrankungen der Venen
}

Seit vielen Jahren schon sind die Erkrankungen der Venen kein Gegenstand mehr in der Zeitschrift „Der Internist“ gewesen. Umso begrüßenswerter ist es, dass nach dem Schwerpunktheft über die Arterienerkrankungen im August 2009 nunmehr das 2. große Thema der Angiologie auf den aktuellen Stand gebracht werden kann. Das Spektrum der Venenerkrankungen reicht von der akuten Venenthrombose über die Varikosis bis hin zur chronischen venösen Insuffizienz. Jede einzelne Erkrankungsgruppe ist dabei so häufig, dass kein praktizierender Arzt nicht regelmäßig damit in Berührung käme.

Wir haben in Deutschland sehr gute Voraussetzungen für eine adäquate Versorgung. Zum einen ist die Aufmerksamkeit der Ärzteschaft gegenüber den Venenerkrankungen im Allgemeinen hoch. Die einzige Ausnahme macht das Krankheitsbild der chronisch-venösen Insuffizienz, die begrifflich und pathophysiologisch nicht ausreichend präsent und daher in der Versorgung ungeordnet ist.

\section{(2) Die Aufmerksamkeit der Ärzteschaft gegenüber den Venenerkrankungen ist im Allgemeinen hoch}

Dagegen wird die akute Venenthrombose als Verdacht häufig geäußert, die entsprechende diagnostische Aufarbeitung erfolgt flächendeckend und ohne wesentlichen Zeitverzug. Die Varikosis wird bereits von den Patienten mit großer Aufmerksamkeit bedacht, ein Mangel an varizentherapeutischen Eingriffen besteht definitiv nicht. Selbst auf dem Gebiet der Thromboseprophylaxe, und hier speziell in der Inneren Medizin, besteht in Deutschland im Unterschied zu anderen Ländern eine hohe Aufmerksamkeit und eine starke Neigung zur medikamentösen Prophylaxe.

Vor diesem Hintergrund ist es umso wichtiger, Mechanismen der Überversorgung und Fehlversorgung zu erkennen, um einem unnötigen Ressourceneinsatz vorzubeugen. Die Autoren der einzelnen Artikel in diesem Schwerpunktheft wurden daher gebeten, sich auf die verfügbare Evidenz und die aktuellen Leitlinien zu beziehen. Problembereiche mit möglicher Überversorgung oder Fehlversorgung sollten als solche benannt werden. Dem entsprechend wurde die grundlegende pathophysiologische Schilderung der einzelnen Krankheitsbilder knapp gehalten, da auf diesem Gebiet in den vergangenen Jahren wenig tief greifende neue Erkenntnisse hinzugekommen sind.

So hoffen wir, den Lesern von „Der Internist", ausgehend von der Expertise der Angiologie als Teilgebiet der Inneren Medizin, eine schnelle und doch gut fundierte Orientierung über die gegenwärtigen diagnostischen und therapeutischen Strategien bei Venenerkrankungen in die Hand gegeben $\mathrm{zu}$ haben, und wünschen dem Heft daher eine breite und wohlwollende Aufnahme.

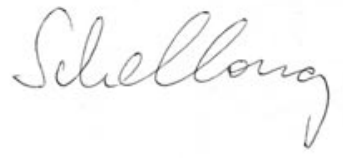

S. Schellong

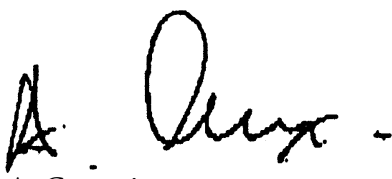

A. Creutzig

\section{Korrespondenzadresse}

Prof. Dr. S. Schellong

Municipal Hospital Friedrichstadt Friedrichstraße 41, 01067 Dresden

schellong-se@khdf.de 